\title{
ON THE ZEROS OF THE BERGMAN FUNCTION IN DOUBLY-CONNECTED DOMAINS
}

\author{
PAUL ROSENTHAL ${ }^{1}$
}

The purpose of this note is to show that every doubly-connected Lu Qi-Keng domain in $C^{1}$ is pseudoconformally equivalent to a disc with the center deleted. This extends a result of M. Skwarczynski [4], who gave an example of a domain $C^{1}$ which is not a Lu Qi-Keng domain. (See definition below.) Our results indicate that, at least in this particular case, there exists a connection between the degree of connectivity of $D$ and zeros of the Bergman function. We use the notation $z=\left(z^{1}, z^{2}, \cdots, z^{n}\right)$ for a point in $D \subset C^{n}$ and $\bar{t}$ for $\left(\bar{t}^{1}, \bar{t}^{2}, \cdots, \bar{t}^{n}\right)$. We denote

$$
D^{*}=\{\bar{t} \mid t \in D\} .
$$

Definitions and theorems relating to the Bergman function can be found in [1]. In what follows, the Bergman function of the domain $D \subset C^{n}$ will be denoted by $K_{D}(z, \bar{t})$.

Definition. A domain $D \subset C^{n}$ is a Lu Qi-Keng domain if the equation $K_{D}(z, \bar{t})=0$ has no solution in $D \times D^{*}$ (see [4]).

Theorem 1. Let $D$ be the ring $0<r<|z|<1$. Then $D$ is not a Lu Qi-Keng domain.

Proof. As was shown by Zarankiewicz in [5], see also $[1$, p. 10],

$$
K_{D}(z, \bar{t})=\frac{1}{\pi z \bar{t}}\left[\wp\left\{\log (z \bar{t}) ; \omega, \omega^{\prime}\right\}+\frac{\eta}{\omega}-\frac{1}{2 \omega^{\prime}}\right],
$$

$\S$ is the Weierstrassian $\wp$-function, $\omega=\pi i, \omega^{\prime}=\log r, 2 \eta$ is the increment of the Weierstrassian $\zeta$-function related to the half-period $\omega$. (We note that since the first half-period $\omega=\pi i$, the value of the 8 function does not depend on the value chosen for $\log (z \bar{t})$.) Using the Legendre equation $\eta \omega^{\prime}-\eta^{\prime} \omega=\pi i / 2\left(\operatorname{real}\left(\omega^{\prime} / i \omega\right)>0\right)$, (1) can be written as

$$
K_{D}(z, \bar{t})=\frac{1}{\pi z \bar{t}}\left[\wp\left(u ; \omega, \omega^{\prime}\right)+\frac{\eta^{\prime}}{\omega^{\prime}}\right],
$$

$u=\log (z \bar{t})$. The function $e^{u}$ maps the period-parallelogram (points

Presented to the Society, April 4, 1968; received by the editors May 1, 1968.

${ }^{1}$ This work was supported in part by Air Force contract AF 1047-66 and AEC contract 326-P22. 
$\operatorname{Re} u=0, \log r^{2}$ excluded) on to the $q$-ring $0<r^{2}<|q|<1, q=z \bar{t}$. Since the doubly periodic function $\delta(u)$ attains every value in the periodparallelogram exactly twice, the function

$$
\eta^{\prime} / \omega^{\prime}+\wp(u)
$$

attains every value in the $q$-ring except for values attained when $u$ is in the segment $\operatorname{Re} u=0,0 \leqq \operatorname{Im} u \leqq \pi$. Consider the boundary of the rectangle with vertices $0, \pi i, \log r+\pi i, \log r$, in the $u$-plane with counterclockwise orientation. On this boundary, $\varnothing$ attains real values increasing monotonically from $-\infty$ to $+\infty$. The function (3) has the same property, and we conclude that the exceptional values of (3) form a closed segment $\left[-\infty, \eta^{\prime} / \omega^{\prime}+\wp(\pi i)\right]$ on the real axis. We infer the Bergman function has a zero in $D \times D^{*}$ if and only if

$$
\eta^{\prime} / \omega^{\prime}+\wp(\pi i)<0 .
$$

We prove next that, for every $0<r<1$, (4) holds. Consider the new pair of primitive half-periods, $\tilde{\omega}=-\log r, \tilde{\omega}^{\prime}=\pi i$. We then obtain

$$
\eta^{\prime} / \omega^{\prime}+\wp(\pi i)=\tilde{\eta} / \tilde{\omega}+\wp(\pi i)
$$

It is known that [2, p. 336],

$$
\begin{aligned}
\frac{\tilde{\eta}}{\tilde{\omega}}+\wp(u)=-\frac{\pi^{2}}{\tilde{\omega}^{2}}\left\{\frac{1}{\left(z-z^{-1}\right)^{2}}+\sum_{m=1}^{m=\infty} \frac{h^{2 m} z^{-2}}{\left(1-h^{2 m} z^{-2}\right)^{2}}\right. & \left.+\sum_{m=1}^{m=\infty} \frac{h^{2 m} z^{2}}{\left(1-h^{2 m} z^{2}\right)^{2}}\right\}
\end{aligned}
$$

Since the right-hand side of (6) for $\tilde{\omega}=-\log r, \tilde{\omega}^{\prime}=\pi i$, and $u=\pi i$ is negative for all $0<r<1$, (4) holds. This completes the proof of Theorem 1.

TheORem 2. Every doubly-connected Lu Qi-Keng domain in $C^{1}$ is pseudoconformally equivalent to a disc with the center deleted.

Proof. Let $D$ be a doubly-connected Lu Qi-Keng domain in $C^{\mathbf{1}}$. It must be pseudoconformally equivalent to one of the three following domains:

(1) a plane with the center deleted,

(2) a ring $(0<r<|z|<1)$,

(3) a disc with the center deleted.

However, the Bergman function for the domain $\{z \mid z \neq 0\}$ is identically zero, and the Bergman function for the ring posseses zeros by 
Theorem 1. Since the class of Lu Qi-Keng domains is invariant under pseudoconformal transformations, (1) and (2) do not occur.

TheOREM 3. For every $k \geqq 3$, there exists a domain $D \subset C^{1}$ of connectivity $k$ which is not a Lu Qi-Keng domain.

Proof. Consider a ring $R$ and let $z_{0}, \bar{t}_{0}$ be such that $K_{R}\left(z_{0}, \bar{t}_{0}\right)=0$. We choose $k-2$ distinct points $z_{1}, \cdots, z_{k-2}$ in $R$ different from $z_{0}$ and $\bar{t}_{0}$. Consider a domain $R_{m}=R-\bigcup_{j=1}^{k-2}\left[z \mid z \in R\right.$ and $\left|z-z_{j}\right| \leqq 1 / m, m$ a positivt integer]. By the Ramadanov Theorem [3], the sequence $K_{R_{m}}\left(z, \bar{t}_{0}\right)$ converges locally uniformly to $K_{D}\left(z, \bar{t}_{0}\right)$ where $D=\bigcup_{m=1}^{\infty} R_{m}$. Since $K_{D}\left(z, \bar{t}_{0}\right) \equiv K_{R}\left(z, \bar{t}_{0}\right)$, we conclude that for sufficiently large $m$, the degree of connectivity of $R_{m}$ is $k$, and by Hurwitz's theorem the function $K_{R_{m}}\left(z, \bar{t}_{0}\right)$ has a zero in $R_{m}$.

\section{REFERENCES}

1. S. Bergman, The kernel function and conformal mapping, Math. Surveys, No. 5, Amer. Math. Soc., Providence, R. I., 1950.

2. H. Hancock, Theory of elliptic functions, 1910.

3. L. Ramadanov, Sur une proprietté de la fonction de Bergman, Acad. Bulgare Sci. (8) 20 (1967), 759-762.

4. M. Skwarczynski, The invariant distance in the theory of pseudo-conformal transformations and the Lu Qi-Keng conjecture, (to appear).

5. K. Zarankiewicz, Über ein numerisches Verfahren zur conformen Abbildung zweifach zusammenhängender Gebiete, Z. Angew. Math. Mech. 14 (1934), 97-104.

\section{STANFORD UnIVERSITY}

